American Journal of Applied Sciences 4 (5): 264-268, 2007

ISSN 1546-9239

(C) 2007 Science Publications

\title{
Turbo-coding of Coherence Multiplexed Optical PPM CDMA System With Balanced Detection
}

\author{
K. Chitra and V.C. Ravichandran \\ Department of ECE, College of Engineering, Anna University, Chennai-600 025, Tamilnadu, India
}

\begin{abstract}
The effects of turbo-coding on the coherence multiplexed optical PPM CDMA systems are shown by evaluating bit error rate (BER) with respect to system parameters such as interleaver length $(K)$, number of simultaneous users $(N)$, code length $(f)$, initial pulsewidth $(t 0)$ and normalized threshold $(\theta)$ by including the effects of thermal noise and shot noise. Turbo-codes increase the number of simultaneous users besides reducing the BER for a decreasing normalized threshold. As the initial pulsewidth reduces from $7 \mathrm{ps}$ to $5 \mathrm{ps}$ the BER reduces from $1.6392 \times 10^{-17}$ to $7.0167 \times 10^{-18}$. For a fixed number of simultaneous users $(N=20)$, an initial pulsewidth, $(t 0=5 \mathrm{ps})$ and a normalized threshold $(\theta=$ $0.84)$, it is seen that the turbo-codes of interleaver length 10 reduces BER in the order $10^{-10}, 10^{-18}$ and $10^{-32}$ for the code length varying as 63,127 and 255 respectively. On comparison with the direct detection it is found that the balanced detection could accommodate an additional number of 8 users at a reduced $\mathrm{BER}$ of $10^{-16}$.
\end{abstract}

Key words: Coherence multiplexing, CDMA networks, turbo-coded PPM, balanced detection

\section{INTRODUCTION}

Optical code division multiple access (OCDMA) technique is an emerging technology which permits multiple users to be multiplexed asynchronously at same wavelength through their unique signature sequences $^{[1]}$. Optical Orthogonal Codes (OOCs) are commonly used as signature sequences for OCDMA communication systems. Presently, turbo-coded optical PPM communication systems are widely employed in OCDMA networks. In addition to a near optimum error correcting performance ${ }^{[2]}$, the turbo-coded PPM systems achieve a better coding gain with reasonable encoding and decoding complexity. A variety of turbo decoding algorithms of varying complexity are implemented in achieving a low bit error $\operatorname{rate}^{[3]}$. However owing to its asynchronous capability, the coherence multiplexed networks are used for high data rates in OCDMA networks ${ }^{[4]}$.

Balanced detection in coherence multiplexing removes signal terms common to both the detectors of high CMRR and produces successful decoding for matched delays and a noise-like jitter signal for unsuccessful decoding. The advantages of a coherence multiplexed CDMA network and turbo-coded optical PPM CDMA system are combined in order to achieve a lower BER and more number of users than that of a direct-detection scheme with APD detectors.

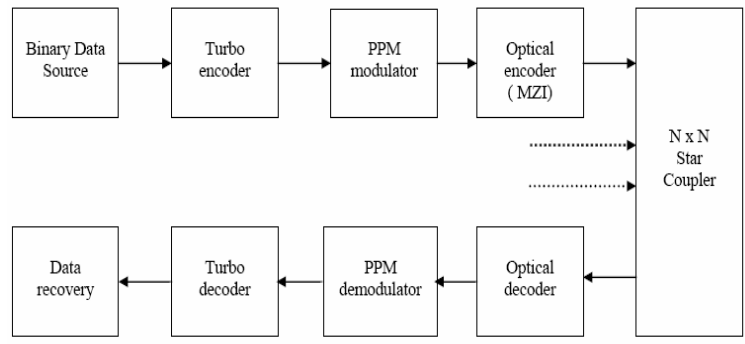

Fig. 1: Block diagram of a coherence multiplexed turbo-coded optical PPM CDMA system

The effects of various noises such as shot noise and thermal noise are also considered. The performance evaluation is carried out using transfer function bounding techniques ${ }^{[5]}$.

System description: The block diagram of the coherence multiplexed turbo-coded optical PPM CDMA system is shown Fig. 1. The binary information of each user is fed to a turbo encoder producing a block-coded sequence of length $K$. A $(1,7 / 5,7 / 5)$ turbo encoder of rate $r=1 / 3$ is considered for analysis with one punctured bit. A multiplexer can be used to transmit the two parity sequences of the turbo encoder alternatively. The information bits of weight $i$ is converted into an output sequence of weight $i+d$, where $d$ is the total weight of the two parity sequences.

Corresponding Author: $\quad$ K.Chitra, Research Scholar, Department of ECE, College of Engineering, Anna University, Chennai- 600 025, Tamilnadu, India 
The turbo-coded output is then passed to an electrical PPM modulator where the coded bit stream is blocked into a symbol of length $\log _{2} M$ bits. A binary PPM signaling format is assumed whereby each turbocoded bit is either placed in zeroth or first slot for an information bit of zero or one respectively.

The optical encoder then encodes the PPM modulated turbo-coded output. Every encoder has a two-arm Mach-Zehnder interferometer (MZI) with a time delay element in one arm and a dispersive element in another arm, which produces signature sequences for CDMA networks. Programmable fiber Bragg gratings ${ }^{[6]}$ can be used for obtaining distinct sequences for many users. The differential delay between any two transmitters must be greater than the source coherence time, $\tau_{c}$, to reduce the crosstalk. For shorter interferometer delays, a broader bandwidth source is to be considered.

The spreaded information sequences from all the asynchronous users are coupled through a $N x N$ star coupler and are connected to balanced receivers which modify the noise power spectral density and improve the signal-to-noise ratio ${ }^{[7]}$. Each receiver has a MZI acting as a decoder and a pair of photodiodes for differential detection ${ }^{[8]}$. The beat noise due to the optical field pairs that travels the same path are cancelled out in the MZI decoder, thereby reducing crosstalk. The optical decoder produces maximum output for authorized users whose encoder and decoder time delays are matched well within the source coherence time. Reprogramming of dispersive elements can also be carried out to match the signature sequences of different pairs of transmitters and receivers.

The PPM demodulator identifies the slot with the highest output as the transmitted sequence. BCJR algorithm ${ }^{[9]}$ is used for producing extrinsic information in the turbo decoder stages. Log-MAP algorithm decodes the signal and recovers the original information bits.

Performance analysis: An upper bound on the turbocoded bit error probability can be derived using transfer function bounding technique. For a PPM channel, the bit error rate depends on the probability of making correct slot decision. The upper bound on turbo-coded bit error probability is given by:

$$
p_{b} \leq \sum_{i=0}^{K} \frac{i}{K} \sum_{d=0}^{2 K}\left(\begin{array}{l}
K \\
i
\end{array}\right) B(i, d) p_{b 1}
$$

where

$$
p_{b 1}=\frac{1}{2} \sum_{i=1}^{N-1}\left(\begin{array}{l}
N-1 \\
i
\end{array}\right) p^{i}(1-p)^{N-1-i}\left[Q\left(\frac{\theta-\mu_{i 0}}{\sigma_{i 0}}\right)+\mathcal{Q}\left(\frac{\mu_{i 1}-\theta}{\sigma_{i 1}}\right)\right]
$$

$$
\begin{aligned}
& p=T_{\sigma} / 2 T_{b} ; \mu_{i 0}=\left(i \sigma^{2}+\lambda\right) ; \mu_{i 1} \\
& =\left(i \sigma^{2}+\lambda+1\right) ; \sigma_{i 0}{ }^{2}=\sigma_{i 1}{ }^{2}=i_{\sigma}^{4}+\lambda+\sigma_{\mathrm{th}}^{2}
\end{aligned}
$$

$\sigma_{\mathrm{th}}^{2}$ is the thermal noise of the detectors. $T_{\sigma}$ is the rms pulsewidth, $T_{b}$ is the bit rate of the individual user, $\sigma^{2}$ is the variance of the Gaussian pulse, $\lambda$ is the mean and variance of the shot noise, $B(i, d)$ is the number of code words generated by an input sequence of input hamming weight $i$ and output hamming weight $d$. The conditional probability, $P(d / i)$, of obtaining a code fragment of weight $d$, for a given input sequence of weight $i$ is ${ }^{[5]}$,

$P(d / i)=\mathrm{t}(\mathrm{K}, \mathrm{i}, \mathrm{d}) / \Sigma_{\mathrm{d}^{\prime}} \mathrm{t}\left(\mathrm{K}, \mathrm{i}, \mathrm{d}^{\prime}\right)$

$=t(K, i, d) /\left(\begin{array}{l}K \\ i\end{array}\right)$

where $K$ is the interleaver length, i.e. the number of information bits and the denominator term in the above equation gives the total number of code words of hamming weight $i$. For a rate $1 / 3$ and $(1,7 / 5,7 / 5)$ turbo encoder, the probability $p\left(d_{1}, d_{2} / i\right)$ that any input sequence of weight $i$ will be mapped into codeword fragments of weights $d_{1}$ and $d_{2}$ is

$P\left(d_{1}, d_{2} / i\right)=\mathrm{P}_{7 / 5}\left(\mathrm{~d}_{1} / \mathrm{i}\right) . \mathrm{P}_{7 / 5}\left(\mathrm{~d}_{2} / \mathrm{i}\right)$

and hence $B(i, d)=\sum_{d_{1}=0}^{K} \sum_{d_{2}=0}^{K} P\left(d_{1}, d_{2} / i\right)$

\section{NUMERICAL RESULTS}

The performance analysis of a coherence multiplexed turbo-coded optical PPM CDMA system is carried out in terms of BER by varying the following system parameters namely number of simultaneous users, normalized threshold, initial pulse width, interleaver length and code length. The BER is computed using Eq.(1) to Eq.(7). Turbo-codes with an interleaver length of 10 and 50 are considered for the numerical analysis. The initial pulsewidth is allowed to vary from 5 ps to $7 \mathrm{ps}$. The code length variation is assumed to be 63,127 and 255 . The bit rate of the individual users is chosen as a typical rate of $1 \mathrm{~Gb} / \mathrm{s}$.

Figure 2 shows that as the number of simultaneous users increases $(N)$, the normalized threshold at which the minimum BER occurs is found to increase and try to converge towards the optimal threshold of 1.0. As $N$ increases from 30 to 35,40 and 50, the BER increases from $3.1526 \times 10^{-20}$ to $2.398 \times 10^{-18}, 9.4706 \times 10^{-17}$ and $3.3074 \times 10^{-14}$ for the threshold varying as 0.80 to 0.82 , 0.84 and 0.86 respectively. 


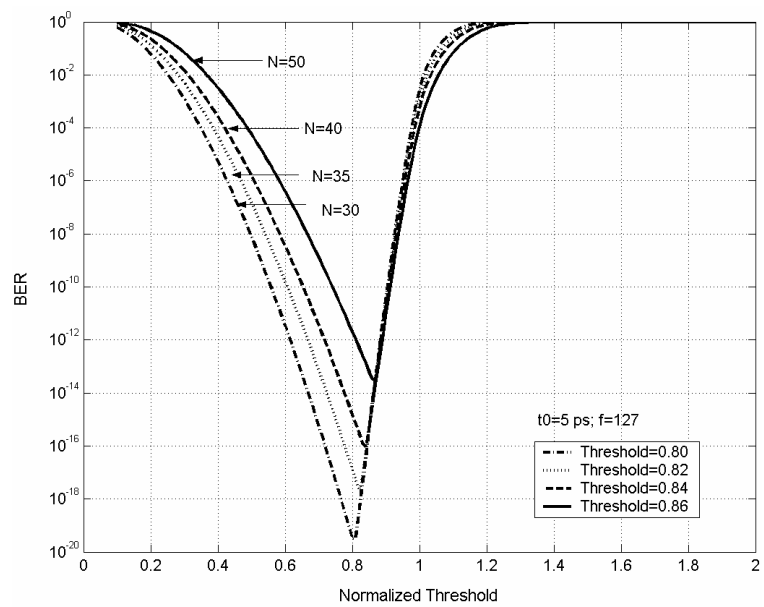

Fig. 2: Normalized Threshold Vs BER for different users

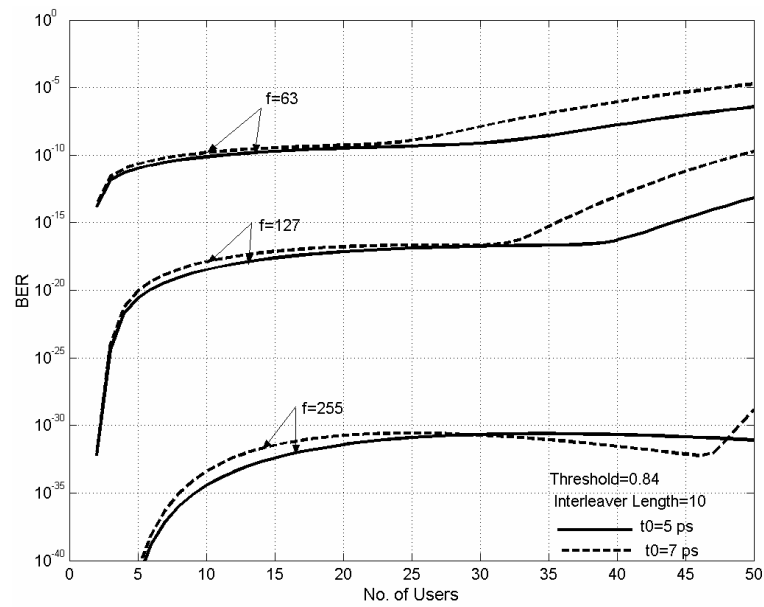

Fig. 3: Number of simultaneous users Vs BER for different code length and initial pulsewidth

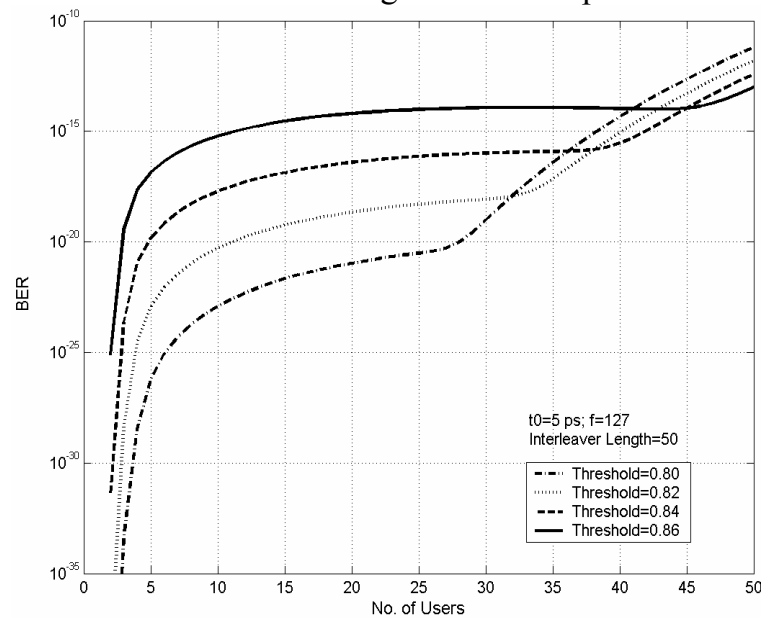

Fig. 4: Number of simultaneous users Vs BER at an Interleaver length of 50

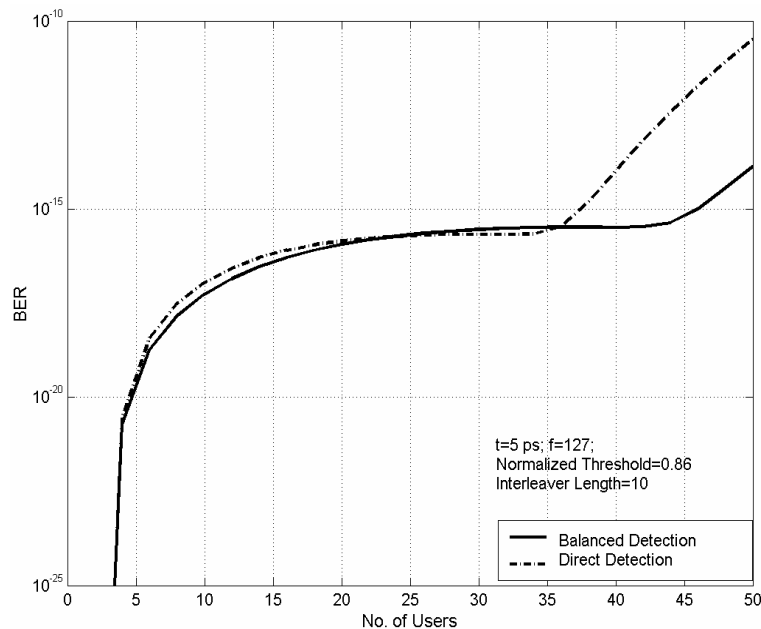

Fig. 5: Number of simultaneous users Vs BER with direct and balanced detection

Turbo-codes with an interleaver length of 10 is applied in Fig. 3 which shows the variation of the BER with the number of simultaneous users at a fixed normalized threshold of 0.84 and the initial pulsewidth varying from 7 ps to $5 \mathrm{ps}$. When the code length is 63, a maximum of 23 users can be accommodated at a specified BER of $4.0852 \times 10^{-10}(t O=5 \mathrm{ps})$ and $7.3375 \times 10^{-}$ ${ }^{10}(t O=7 \mathrm{ps})$ beyond which there is an abrupt increase in the BER. Similarly, when the code length is varied to 127 and 255 the BER reduces to $1.9912 \times 10^{-17}(t O=5 \mathrm{ps})$ and $2.4342 \times 10^{-31}(t O=5 \mathrm{ps})$ respectively with the number of users at that BER increasing from 32 to 34 beyond which there is an abrupt rise. Almost the same BER is maintained for $f=255$ even if the number of users is increased to $48(t 0=5 \mathrm{ps})$. The similar effect for a $t 0$ of $7 \mathrm{ps}$ is also plotted in this figure. The effects of thermal and shot noise are considered in this analysis.

Figure 4 shows the variation of BER as the number of simultaneous users increases at an interleaver length of 50. It is seen that more number of users are supported at a reduced BER for an increasing threshold. For a fixed number of users $(N=26)$ and a threshold of $0.86,0.84,0.82$ and 0.80 the BER is found to reduce as $1.0269 \times 10^{-14}, \quad 8.2109 \times 10^{-17}, \quad 5.6524 \times 10^{-19}$ and $3.7532 \times 10^{-21}$ respectively. Also, it is found that almost the same BER is maintained when the number of users increases to 44, 39, 32 and 28 for the respective threshold variations. The thermal noise and shot noise effects are included in this figure.

Figure 5 compares the performance of the system with direct detection and balanced detection. It is found that the BER is maintained at around $10^{-16}$ for the number of users varying from $19\left(\mathrm{BER}=1.1364 \times 10^{-16}\right)$ to $44\left(\mathrm{BER}=6.1835 \times 10^{-16}\right)$ when balanced detection is 


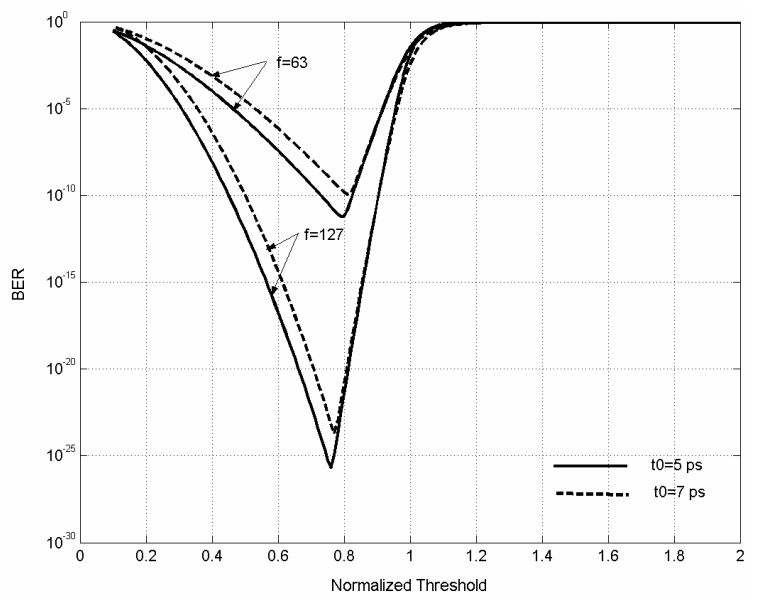

Fig. 6: Normalized Threshold Vs BER for different code length and initial pulsewidth.

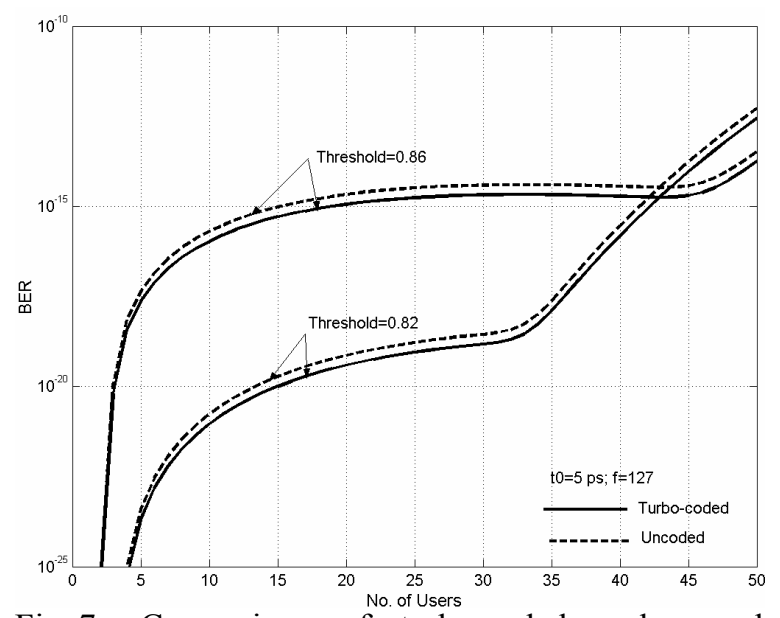

Fig. 7: Comparison of turbo-coded and uncoded system with different threshold

employed. But the BER increases to $1.3756 \times 10^{-14}$ when the number of users increases to 50 . However, when direct detection is employed, it is found that the same BER is maintained for the number of users varying from $19\left(\mathrm{BER}=1.1187 \times 10^{-16}\right)$ to $36\left(\mathrm{BER}=3.3616 \times 10^{-}\right.$ ${ }^{16}$ ) and the BER increases to $3.2536 \times 10^{-11}$ for 50 users. Thus, 25 users are accommodated at a BER of $10^{-16}$ in the case of balanced detection whereas only 17 users are possible for that of direct detection, i.e. an additional number of 8 users are accommodated in balanced detection. The noise effects are ignored in this analysis and the interleaver length is chosen as 10 with $\theta=0.86$.

Figure 6 shows the variation of BER with respect to normalized threshold as the code length is varied from 63 to 127 for a fixed number of 20 users. When the code length is 127 , the threshold is found to increase from 0.76 to 0.77 with BER increasing from $1.9314 \times 10^{-26}(t O=5 \mathrm{ps})$ to $1.9485 \times 10^{-24}(t 0=7 \mathrm{ps})$. For a code length of 63 , the threshold is found to increase from 0.79 to 0.81 with BER increasing from 5.9891 $10^{-}$ ${ }^{12}(t O=5 \mathrm{ps})$ to $1.1038 \times 10^{-10}(t O=7 \mathrm{ps})$. Hence, as the code length increases, the BER reduces.

Figure 7 shows a comparison of uncoded and turbo-coded CDMA system with a normalized threshold of 0.82 and 0.86 . At a BER of $7.2745 \times 10^{-20}$ $(\theta=0.82)$, only 20 users could be supported by an uncoded system whereas 24 users could be supported by a turbo-coded system. However, the same 20 users can be supported by a turbo-coded system with a reduced BER of $3.8738 \times 10^{-20}$. Hence BER in the turbocoded system is reduced by half that of the uncoded system.

\section{CONCLUSION}

From the above analysis it is seen that as the code length increases, BER decreases with turbo-codes applied to coherence-multiplexed PPM CDMA system and the BER is found to reduce to half that of the uncoded system. With an increase in interleaver length of the turbo-code along with an increase in normalized threshold, an extended number of users could be supported at a reduced BER. It was also shown that the balanced detection could accommodate more number of users besides reducing BER compared to direct detection. Hence the balanced detection of turbo coded, coherence-multiplexed PPM modulation is a better solution for a typical data rate of $1 \mathrm{~Gb} / \mathrm{s}$ optical CDMA networks

\section{REFERENCES}

1. Salehi, J.A., 1989. Code division multiple-access techniques in optical fiber networks-Part I: Fundamental principles. IEEE Trans. on Communications, 37: 824-833.

2. Berrou, C. and A. Glavieux, 1996. Near Optimum error correcting coding and decoding: Turbo-codes. IEEE Trans. on Communications, 44: 1261-1271

.3. Kim, J.Y. and H.V. Poor, 2001. Turbo-coded Optical Direct-Detection CDMA System with PPM Modulation. J. Light Wave Tech., 19: 312-322.

4. Griffin, R.A., D.D. Sampson and D.A. Jackson, 1995. Coherence coding for photonic code-division multiple access networks. J. Light Wave Tech., 13: 1826-1837. 
5. Divsalar, D., S. Dolnar, F. Pollara and R.J. McEliece, 1995. Transfer function bounds on the performance of turbo codes. Telecommunications and Data Acquisition Progr. Rep., 42-122: 44-55.

6. Wang L. and A.M.Weiner, 1999. Programmable spectral phase coding of an amplified spontaneous emission light source. Optics Communications, 167: 211-224.

7. Pendock, G.J. and D.D. Sampson, 1997. Noise in coherence multiplexed optical fiber systems. Appl. Optics, 36: 9536-9540.
8. Pendock, G.J. and D.D. Sampson, 1997. Capacity of coherence multiplexed CDMA networks. Optics Communications, 143: 109-117.

9. Bahl, L.R., J. Cocke, F. Jelinek and J. Raviv, 1974. Optimal decoding of linear codes for minimizing symbol error rates. IEEE Trans. Inform. Theory, 20: 284-287. 